a. Consultorios Médicos Pediátricos, Ramos Mejía, Buenos Aires.

b. Department of Mother and Child Health, Hospital Universitario Austral, Pilar, province of Buenos Aires.

c. Department of Nutrition and Diet Therapy, Hospital de Niños de La Plata, La Plata, province of Buenos Aires.

d. Hospital de Niños Ricardo Gutiérrez, Autonomous City of Buenos Aires.

e. Department of Pediatric Gastroenterology and Hepatology, Hospital Italiano de Buenos Aires, Autonomous City of Buenos Aires.

f. Hospital Nacional Alejandro Posadas, El Palomar, province of Buenos Aires.

g. Sanatorio de Niños de

Rosario, Rosario, province of Santa Fe.

h. Department of Pediatric Gastroenterology and Hepatology, Hospital Italiano de Buenos Aires, Autonomous City of Buenos Aires.

i. Sanatorio Anchorena San Martín, Villa Lynch, province of Buenos Aires.

j. Institute of Industrial Dairy Science (National Scientific and Technical Research Council [Consejo Nacional de Investigaciones Científicas y Técnicas, CONICET]/ Universidad Nacional del Litoral [UNL]), School of Chemical Engineering, Universidad Nacional del Litoral, city of Santa Fe. Argentina.

E-mail adress:

María del C. Toca, M.D. mtoca@intramed.net

Funding:

None.

Conflict of interest:

NUTRICIA provided the

place to carry out two face-to-face meetings and an online meeting. The manuscript was written with the collaboration of all authors; NUTRICIA BAGÓ did not interfere with the editorial management or the final article. None of the authors has a business relationship with NUTRICIA BAGÓ. The authors state that their only relationship with NUTRICIA BAGÓ was their participation in conferences and symposiums organized by the company, as in others carried out by other companies.

Received: 8-13-2019 Accepted: 2-7-2020

\title{
Gut ecosystem during infancy: The role of "biotics"
}

\author{
María del C. Toca, M.D. ${ }^{a}$, Fernando Burgos, M.D. ${ }^{b}$, Adriana Fernández, M.D.c, \\ Norberto Giglio, M.D. ${ }^{d}$, Marina Orsi, M.D. ${ }^{e}$, Patricia Sosa, M.D. ${ }^{f}$, Omar Tabacco, M.D. ${ }^{g}$, \\ Florencia Ursino, M.D. ${ }^{h}$, Federico Ussher, M.D. ${ }^{i}$ and Gabriel Vinderola, M.D.
}

\begin{abstract}
In recentyears, theevidence has demonstrated the relevance of the gut microbiota in an individual's health. The dynamics of an early colonization and the establishment of a community of plenty, diverse, and healthy microorganisms from a vaginal delivery and breastfeeding are critical for the development of a healthy immune matrix. The objective of this review is to describe the available evidence on microbiota development in the first year of life and the current possibilities offered by prebiotics, probiotics, symbiotics, and postbiotics during such critical stage of life. Key words: microbiota, breast milk, prebiotics, probiotics, postbiotics.
\end{abstract}

http: / / dx.doi.org/10.5546/ aap.2020.eng.278

To cite: Toca MC, Burgos F, Fernández A, Giglio N, et al Gut ecosystem during infancy: The role of "biotics". Arch Argent Pediatr 2020;118(4):278-285.

\section{INTRODUCTION}

The human microbiota is defined as a complex community of microorganisms living in the body, skin, mouth, nasopharynx, urogenital system, and the gut. ${ }^{1}$ The term microbiome refers to the set of genes contained in such community. ${ }^{1}$ The microbiota is mostly made up of bacteria, but it also includes viruses, fungi, archaea, and bacteriophages. ${ }^{2}$ Its composition varies depending on the genetics and characteristics of the environment offered by the host. ${ }^{3}$ The microbiota is responsible for several digestive and metabolic functions, the production of vitamins, the maintenance of the intestinal barrier, and the regulation of the immune system. ${ }^{1}$ The colonization and establishment of these microorganisms takes place in the first 1000 days of life, from gestation until two years of age. This is a critical period for the development and maturation of the immune system, thus opening a window of opportunities for the development of a healthy microbiota. ${ }^{4}$

The microorganism community experiences a symbiosis and benefits from a stable environment and from nutrients, i.e. carbohydrates not digested by the human gut offered by the intestinal lumen. In turn, the host favors from the fermentation products of such undigested compounds, such as short-chain fatty acids (acetate, propionate, and butyrate), that are metabolized by beneficial bacteria, such as bifidobacteria and lactobacilli, among others, and have multiple functions, including being a source of energy for the intestinal epithelium, reinforcing the intestinal barrier, providing epigenetic effects, and developing immune tolerance. ${ }^{4}$

A group of experts made up of pediatricians and specialists in Nutrition, Pediatric Gastroenterology, and Microbiology met to review the evidence available on microbiota colonization and development in the first year of life, the use of prebiotics, probiotics, symbiotics, and postbiotics in infant formula, and their potential benefits during this critical stage of life.

\section{When does the gut microbiota start developing? Intestinal colonization}

An early massive microbial colonization starts at birth. Vaginal delivery and breastfeeding favor the establishment and development of a healthy neonatal microbiota. The maternal gut microbiota changes its microbiological structure during pregnancy, especially in the third trimester. ${ }^{4,5}$ Its final composition will depend on several factors, including 
diet, body mass index, weight gain during pregnancy, stress, and antibiotic and other drug use, which, in turn, will affect colonization in the newborn infant. ${ }^{6}$ Some evidence suggests that changes in the microbiota are accompanied by changes in intestinal permeability, which promote bacterial translocation for the vertical transmission of microorganisms from mother to child $^{7,8}$ (Figure 1).

From a very early stage, a small number of bacteria (or their compounds) are present in the fetal gut. The characteristics shared by the placental microbiota, the amniotic fluid, and the meconium suggest microbial transfer at the fetalmaternal interface. ${ }^{9,10}$ In spite of these findings, the existence of a fetal microbiota and its role in the development of intestinal colonization are still debatable. ${ }^{11}$

The delivery mode determines the type of microbiota that will develop in the newborn infant. Babies born by vaginal delivery are exposed to vaginal bacteria, mainly Lactobacillus, but also Bacteroides, Prevotella, Parabacteroides, and Escherichia. The maternal gut is a major source of bacteria; $72 \%$ of intestinal bacteria in infants born by vaginal delivery derive from the maternal intestinal bacteria, compared to $41 \%$ in those born by C-section. Therefore, infants born by $\mathrm{C}$-section will be colonized by bacteria associated with the maternal skin and mouth, or the maternal environment in general, predominantly Staphylococcus, Propionibacterium, and Corynebacterium. ${ }^{12-15}$ Infants born by C-section have a less diverse and delayed colonization by Bifidobacterium compared to those born vaginally. ${ }^{16}$

\section{What about the development of a healthy microbiota in the postnatal period? What is the role of breast milk?}

Breast milk is a unique and optimal combination of both macro- and micronutrients, together with several compounds related to immunity, such as regulatory cytokines, chemokines, growth factors, antibodies, bioactive compounds and microorganisms, including oligosaccharides. ${ }^{17}$ Historically, breast milk was considered a sterile fluid, but the paradigm changed in recent years with the detection of the first bacteria not associated with mammary gland infection, e.g., Lactobacillus gasseri. Nowadays, we know that breast milk is the second most important inoculum, a true vehicle for sowing that completes colonization by providing more than $10^{5}$ bacteria per day that come from the maternal gut, an effect of the entero-mammary pathway $^{12,18}$ (Figure 1).

Although multiple studies have shown that the composition of breast milk microbiota varies greatly and depends on genetic, cultural, environmental, and nutritional differences, a recent systematic review of studies that included

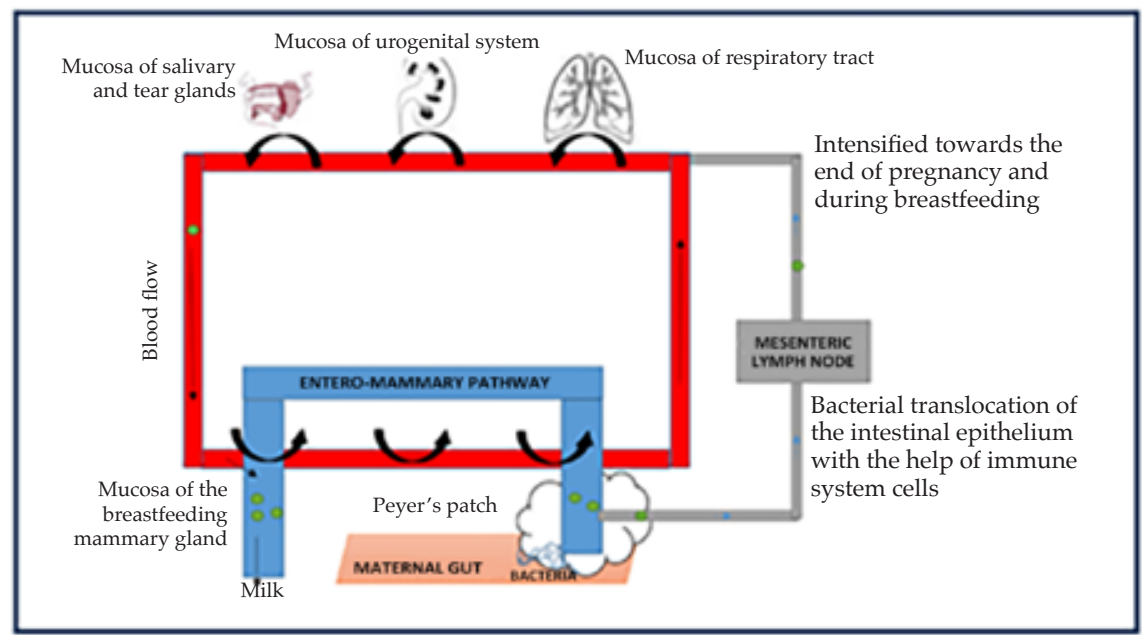

Modified from Rodriguez JM. The Origin of Human Milk Bacteria: Is There a Bacterial Entero-Mammary Pathway during Late Pregnancy and Lactation? Adv Nutr. 2014;5(6):779-84. 
healthy mothers identified Streptococcus and Staphylococcus as the prevalent bacteria, and a lower level of Bifidobacterium and Lactobacillus, regardless of the differences in geographic location or testing methods used for analysis. ${ }^{19}$ Another study showed that, among breastfed infants, $27.7 \%$ of gut microorganisms derived from breast milk and $10.3 \%$, from areolar skin. ${ }^{20}$

The emergence of bacteria is not enough for the development of a healthy microbiota; the environment should be appropriate for their growth and establishment. To that end, breast milk contains bifidogenic factors, currently known as breast milk oligosaccharides, that favor the development of healthy bacteria. These are bioactive compounds with more than 100 different molecular structures.

Breast milk contains diverse oligosaccharides; and lactose, the main carbohydrate, acts as their precursor. They are the third most important compound, after lactose and lipids, at a level of 5-15 g/L. Oligosaccharides are minimally digested in the small intestine; the undigested portion reaches the colon as a metabolic substrate for specific microorganisms, mainly bifidobacteria. ${ }^{21}$

The presence of different oligosaccharides will stimulate the growth of certain bacteria and inhibit the development of others. For example, those known as bifidogenic oligosaccharides stimulate the development of specific bacteria, including Bifidobacterium longum, B. breve, and $B$. bifidum, in the infant gut microbiota, because only these bacteria have enzymes capable of metabolizing such oligosaccharides; therefore, their presence selects the microbiota. ${ }^{2}$

The oligosaccharide content varies from one mother to the other. Such variations have been associated with age, parity, genetics, and environmental factors, including diet, environment, and lifestyle. ${ }^{22}$ The level of oligosaccharides in the milk of mothers who had a preterm infant is higher, and their prebiotic effect has been related to a lower incidence of necrotizing enterocolitis and sepsis. ${ }^{23,24}$ The variations described in oligosaccharide profiles indicate that breast milk provides a tailored nutrition to each infant.

In addition, oligosaccharides not only have a direct impact on microorganisms; they also have indirect effects by altering cellular responses and modulating epithelial apoptosis and cell growth and differentiation. They have also demonstrated their ability to modify the genetic expression of glycocalyx in intestinal surface cells, affect immune cells, and reduce proinflammatory cytokine expression, which would have clinical effects on the development of allergic diseases. ${ }^{25}$ Neonatal colonization is a sensitive and dynamic process directly related to the mode of delivery and breast milk, which is particularly relevant for future health.

\section{How does intestinal colonization affect the immune system maturation?}

Newborn infants have a weak immune response capacity because they are not mature enough yet. Immune maturation depends on the development of innate and adaptive immunity, with a defense response against pathogens and the recognition and symbiosis capacity with tolerance to commensal microorganisms. ${ }^{26}$

The main stimuli for immune maturation are the signals of the microbial environment, which occur in the postnatal period. Interactions between the gut microbiota and the immune system start at birth, when microorganisms provide signals, through endotoxins, fermentation products or compounds typical of bacteria, which encourage the development of gut-associated lymphoid tissue, including Peyer's patches and, at the same time, promote intestinal barrier functions. ${ }^{27,28}$

In turn, the gastrointestinal tract mucosa serves as the most important entry for microorganisms and nutrients, which may lead to an excessive immune stimulation and, thus, generate an inadequate immune activation and intestinal inflammation. From the first weeks of life, thanks to the presence of microorganisms in the intestinal lumen, the immune system will develop a tolerance mechanism. ${ }^{29}$ Based on the development of such ecosystem, the innate and adaptive immune system will be able to differentiate between regulatory responses of tolerance towards the gut microbiota and those towards food proteins, with reduced allergenic and autoimmune responses; in the same way, protective responses to pathogenic bacteria will be observed. ${ }^{30}$ In addition, the protective mechanism of the immune system will restrict the stimulus, covering the epithelial surface with a mucus layer and releasing antimicrobial substances to reconstitute an actual intestinal barrier. ${ }^{26}$

The microbiota promotes the secretion of immunoglobulin A ( $\operatorname{Ig} \mathrm{A}$ ) into the mucosal surface, which plays a critical role to prevent dietary microorganisms and antigens from reaching the epithelial mucus. In turn, the 
intestinal IgA population is key for the selection, development, and maintenance of the gut microbiota, a role played in the first months of life by the IgA present in breast milk, until the infant's own IgA levels increase. ${ }^{31}$ Thus, a gut ecosystem is established where the microbiotahost relationship is mutually beneficial.

\section{What is the role of prebiotics, probiotics,} symbiotics, and postbiotics in infant formula?

In recent years, scientific advances have helped to improve the nutritional compounds and macro- and micronutrients of infant formula to make it more similar to human breast milk. Knowledge of the important function of the gut microbiota in the host's health and of the risk for infants born by $\mathrm{C}$-section who received antibiotics in an early manner and for those who cannot be breastfed forced the advances in preventive nutritional interventions, which will benefit the development of a healthy microbiota. Relevant investigations have shown the advantages of including prebiotics, probiotics, and symbiotics in infant formula and, more recently, the use of postbiotics (Figure 2)..$^{32-36}$

\section{PREBIOTICS}

Prebiotics should meet three main characteristics: a) be resistant to small intestine digestion; $b$ ) be fermented by bacteria in the large intestine; and c) selectively stimulate the activity of one bacterium or a limited number of bacteria in the large intestine, which leads to a beneficial effect. ${ }^{37}$ If breastfeeding is not possible, adding a specific mixture of oligosaccharides to infant formula is a strategy that helps to promote the development of a healthy microbiota. The prebiotics most commonly used in infant formula include fructooligosaccharides (or inulin), which comes from chicory, and galactooligosaccharides, obtained from the fermentation of milk serum lactose.

Several studies have shown that the 90:10 ratio of galactooligosaccharides / fructooligosaccharides (90\% of short-chain galactooligosaccharides and $10 \%$ of long-chain fructooligosaccharides) has a molecular size distribution similar to that of breast milk oligosaccharides. Such combination favors the development of a gut microbiota that is similar to that of breastfed infants, dominated by bifidobacteria and lactobacilli. ${ }^{38}$ The addition of galactooligosaccharides and fructooligosaccharides to infant formula has demonstrated benefits associated with the presence of bifidobacteria, the consistency of stools, and immune system modulation..$^{39}$ More recently, two other oligosaccharides that are added to infant formula -2'-fucosyllactose (2'-FL) and lacto-Nneotetraose (LNnT)-have also been shown to promote bifidobacteria development. ${ }^{40}$

\section{PROBIOTICS}

The following probiotics are used in infant formula: Bifidobacterium animalis subsp. lactis, B. bifidum, B. longum, B. breve, Streptococcus thermophilus, Lactobacillus helveticus, L. rhamnosus, L. reuteri, L. gasseri, L. salivarius, and L. johnsonii. The two most commonly studied probiotics correspond to the Bifidobacterium and Lactobacillus species. Ideally, a probiotic should be well characterized, have no pathogenicity,

FIGURE 2. Definitions: prebiotics, probiotics, symbiotics, and postbiotics

What we know...

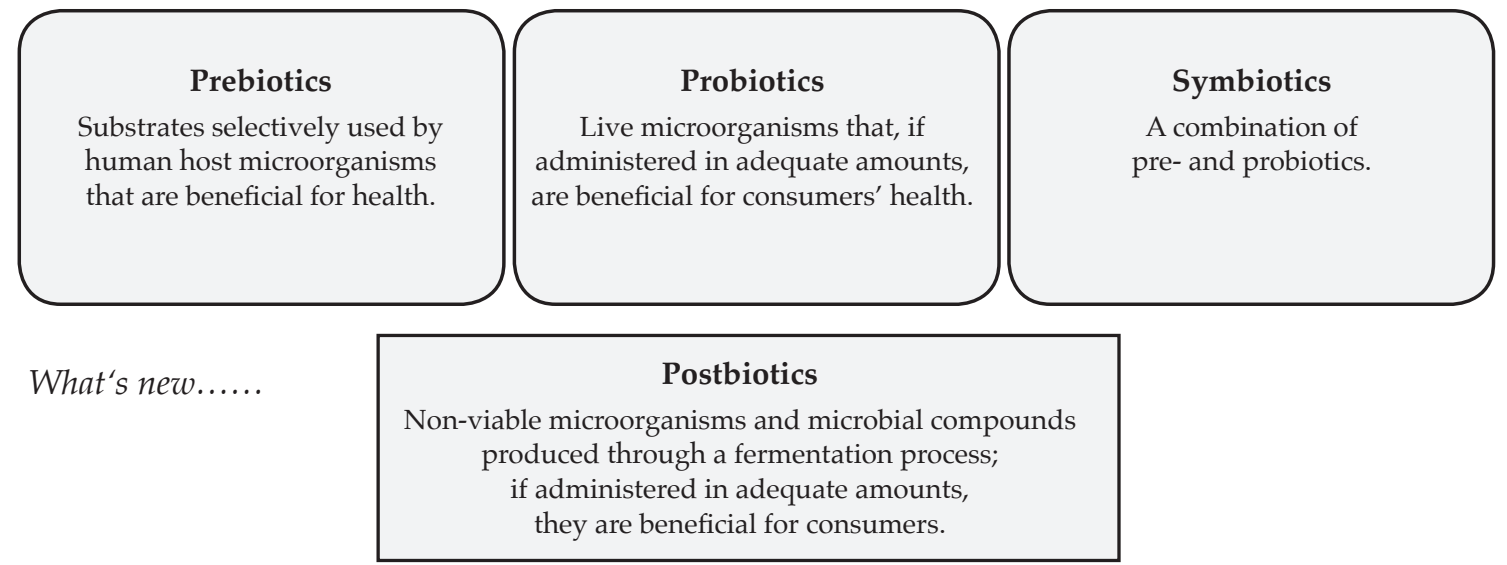


be genetically stable, be robust, and be able to survive in intestinal processing, storage, and transit conditions. ${ }^{40}$

Probiotic activities include improving an altered microbiota, inhibiting pathogens in a competitive manner, having immunomodulatory effects, and regulating intestinal motility. ${ }^{40}$ Not all probiotics are the same. Their actions are specific to each strain and pathology. The results observed in studies for a specific strain cannot be extrapolated to other probiotic strains. It is necessary to use approved quality probiotics with a strong scientific support that warrants their effectiveness and safety. The general effect of adding probiotics to infant formula for the benefit of health still requires confirmation in randomized clinical trials. ${ }^{41}$

\section{SYMBIOTICS}

In the case of symbiotics, the prebiotic compound selectively stimulates colonization and activities of probiotics and other bifidogenic bacteria. Prebiotics may help to improve the survival of probiotics during their passage through the upper gastrointestinal tract, stimulate their growth and/or activate their metabolism. Probiotics and prebiotics may have complementary beneficial effects or a complementary or synergistic effect when combined. ${ }^{40}$ Selectivity is a key consideration in the development of symbiotics; combinations should be selected prudently based on the knowledge of specific patterns for the use of carbohydrates of different bacterial species and strains. $^{40}$

The potential beneficial action of a probiotic intervention, especially Bifidobacterium or Lactobacillus bacteria (common in the infant gut and breast milk), in association with prebiotics (with a bifidogenic effect) for the treatment and prevention of allergic diseases has seized a great deal of attention; recent studies have shown successful results in children with atopic dermatitis. A greater level of protection was observed in the group of infants who were given formula with symbiotics than in the group that received only prebiotic supplementation or the standard formula. ${ }^{42,43}$

\section{POSTBIOTICS}

It has been well established that some bacterial fermentation products and/or the remains of non-viable bacteria have bioactive properties. The fermentation process has been historically used by humans for the preservation of food; nowadays we are aware that many of the known beneficial effects of fermented food are associated with the production of postbiotics. ${ }^{44}$ In addition, it is worth noting that fermentation is also a physiological process that takes place in the gut, where dietary fiber is fermented by the microbiota for the production of vitamins and short-chain fatty acids, among others.

A potential postbiotic production mechanism is that microorganisms (Bifidobacterium or Lactobacillus or Streptococcus) are subjected to a fermentation process (Figure 3), which biochemically transforms the fermented food matrix, in general, milk or dairy substrates. During fermentation, lactose is partially consumed, proteins are partially hydrolyzed, and the metabolites typical of the microorganism used (organic acids, vitamins, exopolysaccharides, bacteriocins, other antimicrobial agents) are synthesized; these molecules will act as postbiotics, even in the absence of the producing microorganism. The microorganism may be eliminated from the fermentation medium by centrifugation or filtration, or it may remain inactive in such medium. The in situ inactivation of the microorganism used for fermentation is achieved through heat treatment, which may be accompanied by a dehydration process, such as lyophilization or spray drying. ${ }^{45}$

Table 1 shows the different types of postbiotics. Several postbiotic mechanisms of action are common to probiotics because the former derive, in most cases, from the latter. Postbiotics act by means of their antimicrobial ability, enzymatic ability to contribute to intestinal digestion, antiinflammatory ability, immunostimulatory ability or the reinforcement of the intestinal epithelial barrier. ${ }^{46,47}$

The main advantage of using postbiotics is that, since they are not microbial cells that should be kept alive, maintaining the cold chain is not a requirement for product conservation, and this makes it possible to add postbiotics to many food matrices and maintain their stability, which sets them apart from probiotics. Finally, postbiotics have other properties that may be considered an advantage: known chemical structures and easier determination of safe doses. ${ }^{48}$

\section{Final comment}

In recent years, the importance of maternal gut microbiota and its impact on the development of the infant's microbiota have 


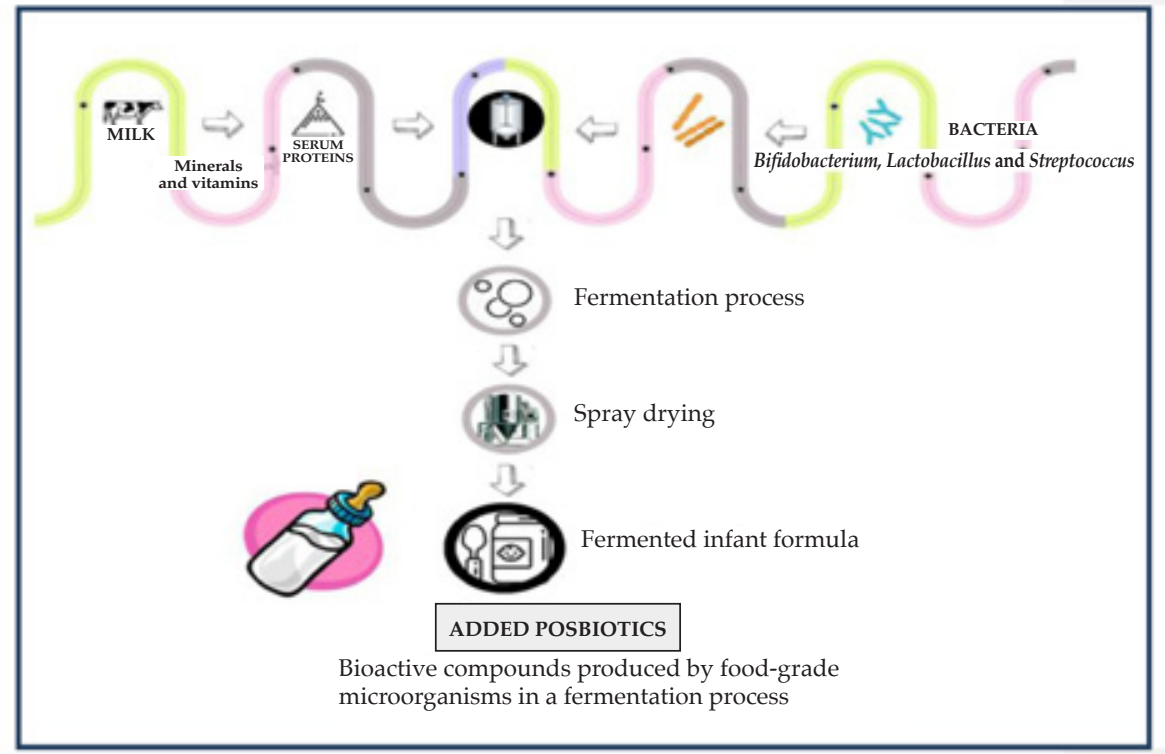

Modified from Seppo Salminen, Hania Szajewska, and Jan Knol. The Biotics Family in Early Life. Editorial John Wiley and Sons Ltd, The Atrium, Southern Gate, Chichester, West Sussex PO19 8SQ, United Kingdom. 2019.

TABLE 1. Types of postbiotics

1) Non-viable cells.

2) Intracellularly synthesized metabolites secreted into the medium during the fermentation process: lactic acid and other organic acids, exopolysaccharides, bacteriocins, enzymes, surfactants, antioxidants, short-chain fatty acids.

3) Biomolecules released into the medium through an extracellular enzymatic activity: peptides, galactooligosaccharides.

4) Compounds released by cell lysis: deoxyribonucleic acid (DNA), ribonucleic acid (RNA), cell wall fragments, S-layer proteins.

been demonstrated based on the evidence. An early and healthy colonization from vaginal delivery and breastfeeding is the role model for the establishment of a gut ecosystem where the microbiota-host relationship is mutually beneficial. The scientific evidence has shown that using prebiotics, probiotics, symbiotics, and postbiotics may restore lost or damaged gut microbiota functions. Based on such knowledge, it would be an interesting challenge to continue studying this fascinating phenomenon to find new strategies for the promotion of pediatric health and the prevention of potential diseases.

\section{Acknowledgments}

We would like to thank NUTRICIA BAGÓ for having made the meetings possible.

\section{REFERENCES}

1. Young VB. The role of the microbiome in human health and disease: an introduction for clinicians. BMJ. 2017; 356:j831.

2. Milani CM, Duranti S, Bottacini F, Casey E, et al. The First Microbial Colonizers of the Human Gut: Composition, Activities, and Health Implications of the Infant Gut Microbiota. Microbiol Mol Biol Rev. 2017; 81(4):e00036-17.

3. Yatsunenko T, Rey FE, Manary MJ, Trehan I, et al. Human gut microbiome viewed across age and geography. Nature. 2012; 486(7402):222-7.

4. Selma-Royo M, Tarrazó M, García-Mantrana I, GómezGallego C, et al. Shaping Microbiota During the First 1000 Days of Life. Adv Exp Med Biol. 2019; 1125:3-24.

5. JiménezE,Marín M,Martín R, Odriozola J, etal.Is meconium from healthy newborns actually sterile? Res Microbiol. 2008; 159(3):187-93.

6. Mulligan C, Friedman J. Maternal modifiers of the infant gut microbiota - metabolic consequences. J Endocrinol. 2017; 235(1): R1-12.

7. Neu J. The microbiome during pregnancy and early postnatal life. Semin Fetal Neonatal Med. 2016; 21(6):373-9. 
8. Rodriguez JM. The Origin of Human Milk Bacteria: Is There a Bacterial Entero-Mammary Pathway during Late Pregnancy and Lactation? Adv Nutr. 2014; 5(6):779-84.

9. Aagaard K, Ma J, Antony KM, Ganu R, et al. The placenta harbors a unique microbiome. Sci Transl Med. 2014; 6(237):237ra65.

10. Collado M, RautavaS, Aakko J, Isolauri E, et al. Human gut colonisation may be initiated in utero by distinct microbial communities in the placenta and amniotic fluid. Sci Rep. 2016; 6:23129.

11. Perez-Muñoz ME, Arrieta MC, Ramer-Tait AE, Walter J. A critical assessment of the "sterile womb" and "in utero colonization" hypotheses: implications for research on the pioneer infant microbiome. Microbiome. 2017; 5(1):48.

12. Ruemmele FM, Bier D, Marteau ZP, Rechkemmer SG, et al. Clinical Evidence for Immunomodulatory Effects of Probiotic Bacteria. J Pediatr Gastroenterol Nutr. 2009; 48(2):126-41.

13. Dominguez-Bello M, Costello E, Contreras M, Magris M, et al. Delivery mode shapes the acquisition and structure of the initial microbiota across multiple body habitats in newborns. Proc Natl Acad Sci U S A. 2010; 107(26):11971-5.

14. Biasucci G, Rubini M, Riboni S, Morelli L, et al. Mode of delivery affects the bacterial community in the newborn gut. Early Hum Dev. 2010; 86(Suppl 1):13-5.

15. Bäckhed F, Roswall J, Peng Y, Fenq Q, et al. Dynamics and stabilization of the human gut microbiome during the first year of life. Cell Host Microbe. 2015; 17(5):690-703.

16. Rutayisire E, Huang K, Liu Y, Tao F. The mode of delivery affects the diversity and colonization pattern of the gut microbiota during the first year of infants' life: a systematic review. BMC Gastroenterol. 2016; 16(1):86.

17. Ballard $O$, Morrow A. Human milk composition: nutrients and bioactive factors. Pediatr Clin North Am. 2013; 60(1):4974 .

18. Soto A, Martín V, Jiménez E, Mader Z, et al. Lactobacilli and bifidobacteria in human breast milk: influence of antibiotherapy and other host and clinical factors. J Pediatr Gastroenterol Nutr. 2014; 59(1):78-88.

19. Fitzstevens J, Smith K, Hagadorn J, Caimano M, et al. Systematic Review of the Human Milk Microbiota. Nutr Clin Pract. 2017; 32(3):354-64.

20. Pannaraj PS, Li F, Cerini C, Bender JM, et al. Association between breast milk bacterial communities and establishment and development of the infant gut microbiome. JAMA Pediatr. 2017; 171(7):647-54.

21. Bode L. The functional biology of human milk oligosaccharides. Early Hum Dev. 2015; 91(11):619-22.

22. Smilowitz JT, Lebrilla CB, Mills DA, GermanJB, et al. Breast milk oligosaccharides: structure-function relationships in the neonate. Annu Rev Nutr. 2014; 34:143-69.

23. Azad MB, Robertson B, Atakora F, Becker AB, et al. Human Milk Oligosaccharide Concentrations Are Associated with Multiple Fixed and Modifiable Maternal Characteristics, Environmental Factors, and Feeding Practices. J Nutr. 2018; 148(11):1733-42.

24. Bering SB. Human Milk Oligosaccharides to Prevent Gut Dysfunction and Necrotizing Enterocolitis in Preterm Neonates. Nutrients. 2018; 10(10):E1961.

25. Eiwegger T, Stahl B, Haidl P, Schmitt J, et al. Prebiotic oligosaccharides: in vitro evidence for gastrointestinal epithelial transfer and immunomodulatory properties. Pediatr Allergy Immunol. 2010; 21(8):1179-88.

26. Hooper L, Littman DR, Macpherson AJ. Interactions between the microbiota and the immune system. Science.
2012; 336(6086):1268-73.

27. Pietrobelli A, Agosti M, MeNu Group. Nutrition in the First 1000 Days: Ten Practices to Minimize Obesity Emerging from Published Science. Int J Environ Res Public Health. 2017; 14(12):E1491.

28. Min YW, Rhee PL. The Role of Microbiota on the Gut Immunology. Clin Ther. 2015; 37(5):968-75.

29. Kamada N, Núñez G. Regulation of the Immune System by the Resident Intestinal Bacteria. Gastroenterology. 2014; 146(6):1477-88.

30. Wang J, Sampson HA. Food allergy. J Clin Invest. 2011; 121(3):827-35.

31. Dollé L, Tran H, Etienne-Mesmin L, Chassaing B. Policing of gut microbiota by the adaptive immune system. BMC Med. 2016; 14:27.

32. Gibson GR, Roberfroid MB. Dietary modulation of the human colonic microbiota: introducing the concept of prebiotics. J Nutr. 1995; 125(6):1401-12.

33. Hill C, Guarner F, Reid G, Gibson G, et al. Expert consensus document. The International Scientific Association for Probiotics and Prebiotics consensus statement on the scope and appropriate use of the term probiotic. Nat Rev Gastroenterol Hepatol. 2014; 11(8):506-14.

34. Mosca F, Gianni ML, Rescigno M. Can Postbiotics Represent a New Strategy for NEC? Adv Exp Med Biol. 2019; 1125:3745.

35. Cicenia A, Scirocco A, Carabotti M, Pallotta L, et al. Postbiotic activities of lactobacilli-derived factors. J Clin Gastroenterol. 2014; 48(Suppl 1):S18-22.

36. Tsilingiri K, Rescigno M. Postbiotics: what else? Benef Microbes. 2013; 4(1):101-7.

37. Roberfroid M. Prebiotics: the concept revisited. J Nutr. 2007; 137(3 Suppl 2):S830-7.

38. Moro G, Minoli I, Mosca M, Fanaro S, et al. Dosage-related bifidogenic effects of galacto and fructooligosaccharide in formula-fed term infants. J Pediatr Gastroenterol Nutr. 2002; 34(3):291-5.

39. Veereman-Wauters G, Staelens S, Van de Broek H, Plaskie $\mathrm{K}$, et al. Physiological and bifidogenic effects of prebiotic supplements in infant formulae. J Pediatr Gastroenterol Nutr. 2011; 52(6):763-71.

40. Salminen S, Szajewska H, Knol J (eds.). The Biotics Family in Early Life. United Kingdom: Wiley; 2019.

41. Thomas DW, Greer FR; American Academy of Pediatrics Committee on Nutrition; American Academy of Pediatrics Section on Gastroenterology, Hepatology, and Nutrition. Probiotics and prebiotics in pediatrics. Pediatrics. 2010; 126(6):1217-31.

42. Sevelsted A, Stokholm J, Bonnelykke K, Bisgaard H. Cesarean section and chronicimmune disorders. Pediatrics. 2015; 135(1):e92-8.

43. Young R. Prebiotics and Probiotics in Pediatric Diarrheal Disorders. In: Sungsoo Cho S, Finocchiaro ET (eds.). Handbook of Prebiotics and Probiotics Ingredients. Health benefits and Food Applications. Boca Raton, FL: CRC Press; 2010.

44. Chilton SN, BurtonJP, Reid G. Inclusion of fermented foods in food guides around the world. Nutrients. 2015; 7(1):390404.

45. Dunand E, Burns P, Binetti A, Bergamini C, et al. Postbiotics produced at laboratory and industrial level as potential functional food ingredients with the capacity to protect mice against Salmonella infection. J Appl Microbiol. 2019; 127(1):219-29.

46. Martin L, Granier A, Lemoine R, Dauba A, et al. Bifidobacteria BbC50 fermentation productsinduce Human 
Cd4+ Regulatory T cells with antigen-specific activation and bystander suppression. Eur J Inflamm. 2014; 12(1):16776.

47. Vinderola G, Matar C, Perdigón G. Milk fermentation products of L. helveticus R389 activate calcineurin as a signal to promote gut mucosal immunity. BMC Immunol. 2007; 8:19 AM.

48. Aguilar-Toalá JE, García-Varela R, García HS, Mata-Haro $\mathrm{V}$, et al. Postbiotics: An evolving term within the functional foods field. Trends Food Sci Technol. 2018; 75:105-14. 